\title{
Estimation of Muscle Forces and Joint Moments Using a Forward-Inverse Dynamics Model
}

\author{
THOMAS S. BUCHANAN ${ }^{1}$, DAVID G. LLOYD ${ }^{2}$, KURT MANAL $^{1}$, and THOR F. BESIER ${ }^{2}$ \\ ${ }^{I}$ Center for Biomedical Engineering Research, Department of Mechanical Engineering, University of Delaware, Newark, \\ DE; and ${ }^{2}$ School of Human Movement and Exercise Science, University of Western Australia, AUSTRALIA
}

\begin{abstract}
BUCHANAN, T. S., D. G. LLOYD, K. MANAL, and T. F. BESIER. Estimation of Muscle Forces and Joint Moments Using a Forward-Inverse Dynamics Model. Med. Sci. Sports Exerc., Vol. 37, No. 11, pp. 1911-1916, 2005. Purpose: This paper presents a forward dynamic neuromusculoskeletal model that can be used to estimate and predict joint moments and muscle forces. It uses EMG signals as inputs to the model, and joint moments predicted are verified through inverse dynamics. The aim of the model is to estimate or predict muscle forces about a joint, which can be used to estimate the corresponding joint compressive forces, and/or ligament forces in healthy and impaired subjects, based on the way they activate their muscles. Methods: The estimation of joint moments requires three steps. In the first step, muscle activation dynamics govern the transformation from the EMG signal to a measure of muscle activation-a time-varying parameter between 0 and 1 . In the second step, muscle contraction dynamics characterize how muscle activations are transformed into muscle forces. The final step requires a model of the musculoskeletal geometry to transform muscle forces to joint moments. Each of these steps involves complex, nonlinear relationships. Results: An application is provided to demonstrate how this model can be used to study the forces in the healthy ankle during dynamometer trials and during gait. The model-predicted estimates of joint moment were found to match experimentally determined values closely. Conclusion: Neuromusculoskeletal models that use EMG as inputs can be employed to accurately estimate joint moments. The muscle forces predicted from these models can be used to better understand tissue loading in joints, and to provide in vivo estimates of tensile ligament forces and compressive cartilage loads during dynamic tasks. This tool has great potential for aiding in the study of injury mechanisms in sports.
\end{abstract} Key Words: EMG, HILL MODEL, TENDON, PENNATION ANGLE, ANKLE

$\mathrm{T}$ There are two traditional modeling approaches to studying the biomechanics of human movement: forward dynamics and inverse dynamics. Either approach can be used to determine joint kinetics (e.g., estimate joint moments during movements), but they each have limitations if used to study muscle contributions to specific sports or exercises.

In forward dynamics, one begins with a measure or estimate of neural command. This is then transformed through a three-step process to obtain joint moments (Fig. 1). Muscle activation dynamics transform the neural signal to a measure of muscle activation, which is a time varying parameter between zero and one. Muscle contraction dynamics transform the muscle activations into muscle forces. Finally, musculoskeletal geometry transforms muscle forces to joint moments. Once the joint moments are determined, the equations of motion allow the joint moments to be transformed

\footnotetext{
Address for correspondence: Thomas S. Buchanan, Center for Biomedical Engineering Research, Department of Mechanical Engineering, University of Delaware, Newark, DE 19716; E-mail: buchanan@me.udel.edu.

Submitted for publication December 2004.

Accepted for publication June 2005.
}

0195-9131/05/3711-1911/0

MEDICINE \& SCIENCE IN SPORTS \& EXERCISE E

Copyright (C) 2005 by the American College of Sports Medicine

DOI: 10.1249/01.mss.0000176684.24008.6f into joint movements. Each of these steps involves complex, nonlinear relationships.

In inverse dynamics, the problem is approached from the opposite end (Fig. 1). Here we begin by measuring position and the external forces acting on the body. For example, in gait analysis, the position of tracking targets attached to the subjects' limbs are recorded using a camera-based video system and the external forces are recorded using a force platform. The tracking targets on adjacent limb segments are used to calculate relative position and orientation of the segments, and from these, joint angles are calculated. These data are differentiated to obtain velocities and accelerations. The accelerations and the information about other forces exerted on the body (e.g., the recordings from a force plate) can be input to the equations of motion to compute the corresponding joint reaction forces and moments. If the musculoskeletal geometry is included, muscle forces could then, in theory, be estimated from the joint moments. However, the problems of cocontraction and redundancy make this difficult.

Although widely used, there are several disadvantages to using inverse dynamics if one is interested in examining muscle function in sports and exercise. First, the moments and resultant joint reaction forces are net values. For example, if a person activates his hamstrings, generating a $25-\mathrm{N} \cdot \mathrm{m}$ flexion moment, and at the same time activates the quadriceps, generating a $20-\mathrm{N} \cdot \mathrm{m}$ extension moment, the inverse dynamics method (if it is perfectly accurate) will 


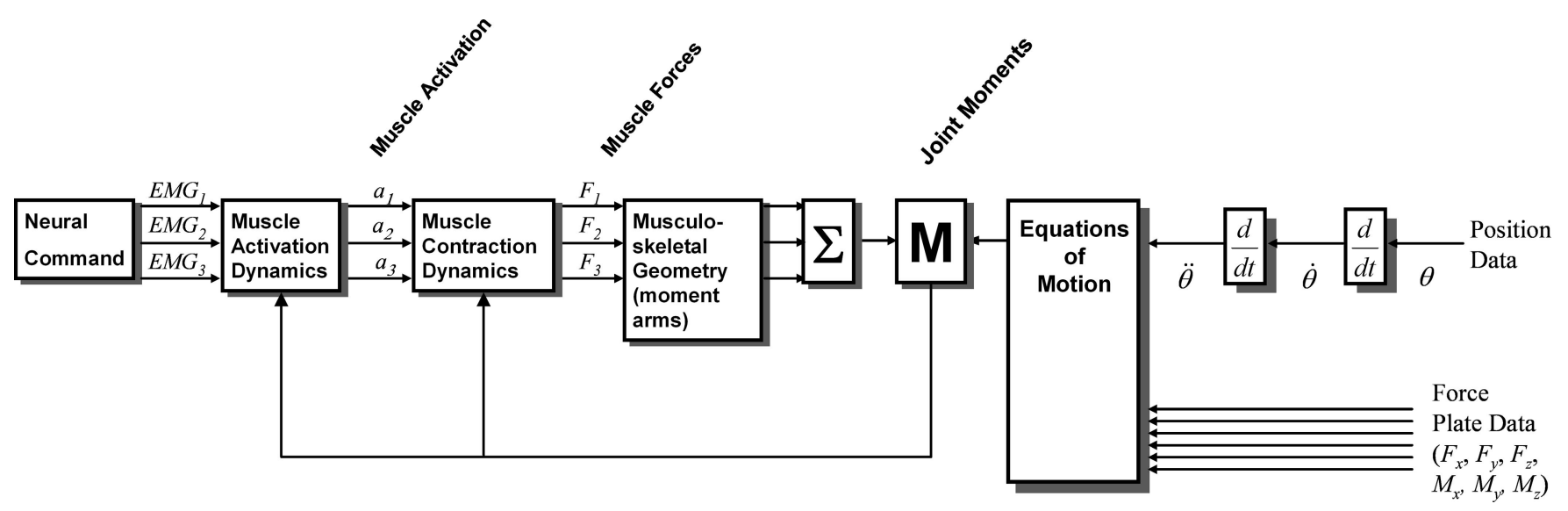

FIGURE 1-Hybrid forward-inverse dynamics approach to estimating joint moments. Beginning at the left, the forward dynamics approach starts with the neural command and then uses muscle activation dynamics, muscle contraction dynamics, and musculoskeletal geometry to estimate the joint moments. These joint moments are also estimated using inverse dynamics (beginning at the right). The error in the joint moment is used to adjust the model parameters during the training phase of the model.

yield a net knee flexion moment of $5 \mathrm{~N} \cdot \mathrm{m}$. This is very important because cocontraction of muscles is very common; yet this approach is widely used to estimate muscular contributions. Second, another limitation of the inverse dynamics approach occurs when one tries to estimate muscle forces. Because there are multiple muscles spanning each joint, the transformation from joint moment to muscle forces yields a large number of possible solutions and cannot be readily determined. Finally, if one wishes to examine muscle activations, there is no current model available that will do this inverse transformation from muscle forces, if muscle forces could be estimated in the first place. Thus, inverse dynamics is not a good method to use if one wishes to include neural activation in the model.

Forward dynamics approaches also have limitations. First, they require estimates of muscle activation. Either one must begin with experimentally based measures of EMG or use a mathematically based optimization approach to estimate neural command. EMG-driven models of varying complexity have been used recently to estimate moments about the knee $(12,13,18)$, the lower back $(17,21)$, the wrist $(3)$, and the elbow (16). The optimization approach, although growing in use $(1,19)$, is limited for studying pathology because it does not include any measure of how a particular person activates his muscles. That is, the cost function chosen may not reflect the actual muscle activation pattern used by a person, especially one with motor impairments.

Second, the transformation from muscle activation to muscle force is difficult, as it is not completely understood. Most models of this are based on phenomenological models derived from A. V. Hill's classic work (10). Another difficulty is that of determining muscle-tendon moment arms and lines of action. These are difficult to measure in cadavers, and even harder to determine with accuracy in a living person. Finally, estimations of joint moments are prone to error because it is difficult to obtain accurate estimates of force from every muscle, and there is often no way to verify whether the forces predicted are correct.

In this paper we present a hybrid approach that uses an EMG-driven forward dynamics model to estimate muscle forces and, from those, joint moments at the ankle. The joint moments were also estimated using inverse dynamics and the parameters in the model were adjusted so that the joint moments from the forward and inverse approaches were in close agreement. Once the parameters were adjusted, the model was used to estimate muscle forces about the ankle for novel tasks. From knowledge of all of the muscle forces about the ankle and their lines of action, one can estimate compressive forces (i.e., in cartilage) and tensile forces in soft tissues (i.e., ligaments).

\section{METHODS}

In this section, the theoretical background of the model will first be presented, followed by a description of the data acquisition procedures.

Muscle activation dynamics. The transformation from EMG to muscle activation is complex. After collection, the initial processing of the EMG signal requires that the signals be rectified and low-pass filtered (fourth-order Butterworth filter, cutoff frequency $8 \mathrm{~Hz}$ ) to form a linear envelope. The signals are then normalized by dividing them by the EMG obtained during a maximal voluntary contraction. The resultant signal is now smooth, between 0 and 1 in magnitude. However, it is still lacking as a representation of muscle activation for three reasons. First, the signal is out of phase with muscle force, as there is a delay between the time of the EMG and that of the corresponding force generation that must be taken into account. This electromechanical delay has been reported to range from $10 \mathrm{~ms}$ to about $100 \mathrm{~ms}$ (4).

Second, muscle activation is known to be a function of its recent history (24). That is, the transformation can be modeled as a second-order differential equation. This has be represented in a discretized form $(11,21)$ as:

$$
\mathrm{u}(\mathrm{t})=\alpha \mathrm{e}(\mathrm{t}-\mathrm{d})-\beta_{1} \mathrm{u}(\mathrm{t}-1)-\beta_{2} \mathrm{u}(\mathrm{t}-2)
$$

where $\mathrm{d}$ is the electromechanical delay and $\alpha, \beta_{1}$, and $\beta_{2}$ are the coefficients that define the second-order dynamics. These parameters $\left(\mathrm{d}, \alpha, \beta_{1}\right.$, and $\left.\beta_{2}\right)$ map the normalized EMG values, e $(\mathrm{t})$, to the neural activation values, $\mathrm{u}(\mathrm{t})$. This 
filter should have unit gain so that neural activation does not exceed 1 , and to ensure this, the following equation has to be met: $\alpha-\beta_{1}-\beta_{2}=1$. In addition, there are several constraints that should be included if the equations are to be stable (see Buchanan et al. (2) for a discussion of equation stability criteria).

Finally, it has been shown that isometric EMG is not necessarily linearly related to muscle force $(8,22,26)$. This nonlinearity is not characterized by the $\mathrm{u}(\mathrm{t})$ term above. For example, Woods and Bigland-Ritchie (22) showed that whereas some muscles have very linear isometric EMGforce relationships, the relationships for other muscles is nonlinear, especially at lower forces (up to about 30\%). Manal and Buchanan (14) have characterized this as a logarithmic relationship for forces less than $30 \%$ and as a linear one for higher force levels.

$$
\begin{array}{ll}
\mathrm{a}(\mathrm{t})=\mathrm{d} \ln (\mathrm{cu}(\mathrm{t})+1), & 0 \leq \mathrm{u}(\mathrm{t})<\sim 0.3 \\
\mathrm{a}(\mathrm{t})=\mathrm{mu}(\mathrm{t})+\mathrm{b}, & \sim 0.3 \leq \mathrm{u}(\mathrm{t})<1
\end{array}
$$

where $u(t)$ is the neural activation (from above) and $a(t)$ is the muscle activation. The coefficients $\mathrm{c}, \mathrm{d}, \mathrm{m}$, and b can be solved for simultaneously and reduced to a single parameter, A (14).

Muscle contraction dynamics. A Hill-type muscle model is used to estimate the force that can be generated by the contractile element of the muscle fiber, with the general form of the function given by:

$$
F^{\mathrm{m}}(\mathrm{t})=f(\mathrm{v}) f(\mathrm{l}) \mathrm{a}(\mathrm{t}) F_{\mathrm{o}}^{\mathrm{m}}
$$

where $F^{\mathrm{m}}$ is the time varying muscle fiber force, $f(\mathrm{v})$ is the normalized velocity-dependent fiber force (i.e., the forcevelocity relationship), $f(1)$ is the normalized length-dependent fiber force (i.e., the length-tension relationship), a(t) is the time-varying muscle activation (as described above), and $F_{\mathrm{o}}^{\mathrm{m}}$ is the maximum isometric muscle fiber force.

Each of these functions is, of course, somewhat complex. For example, the normalized length-tension relationship, $f(1)$, is classically characterized only for muscles at maximal isometric force. However, it has been reported that optimal fiber lengths - the fiber lengths at which the forces peakincrease as activation decreases (7). This creates a skewing of the length-tension relationship that has been mathematically characterized by Lloyd and Besier (11).

Another complexity that must be accounted for is the tendon stiffness. As the muscle contracts, the tendon is stretched, which loads the muscle, causing it to lengthen. Hence, muscle force must be solved for iteratively, as described by Buchanan et al. (2).

Pennation angle should also be taken into account, as it affects the angle of the force transmission. A model that assumes that muscle has constant thickness and volume as it contracts has been shown to characterize the pennation angle behavior well (20).

Musculoskeletal geometry. To compute both the length and the moment arm for a musculotendonous unit, a musculoskeletal model is required. These models must account for the way musculotendon lengths and moment arms change as a function of joint angles, and include information about the geometry of the bones and the complex relationships associated with joint kinematics $(5,6)$.

Once all of the muscle forces are computed and their corresponding moment arms are estimated, their contributions to the joint moment can be found by multiplication. If this is done for all of the muscles at a particular joint, the corresponding joint moment can be estimated by summing these values.

Model parameters. There are several physiological parameters required by the models. Muscle moment arms and lengths were calculated using the model of Delp et al. (6). Optimal fiber length, tendon slack length, and pennation angle are measured from cadaver studies (23). Of these three parameters, tendon slack length is the most difficult to measure, but it can be approximated using a numerical method (15). Maximal muscle force can be estimated from measurements of physiological cross-sectional areas of muscles, which can then be scaled uniformly to match maximal joint strength measures made on a dynamometer (12).

Other parameters in the model cannot be readily determined, such as those that describe muscle activation dynamics. These parameters must be determined mathematically by comparing the estimated joint moments from the forward dynamics calculations with the inverse dynamics determination of the joint moments. These should be the same, and model parameters can be adjusted until the error between these two is minimized. This is done using nonlinear optimization. We use a simulated annealing algorithm, as it is designed to reduce the chances of converging to a local minimum.

Once the model's parameters are adjusted to minimize the error, the model can be used to predict the joint moments and muscle forces during novel tasks - tasks that were not used to minimize the model error. The accuracy of the model can be determined by comparing its predicted estimates of joint moments with the experimentally determined values.

Parameters are adjusted to tune or calibrate the model for each subject (Fig. 2). The exact number of parameters that are to be adjusted using this approach can vary from about one to six per muscle (9). In the application that follows for the ankle, 22 parameters were adjusted. These included the coefficients in equations 1 and 2, as well as the values for each muscle's optimal fiber length (the length at which the length-tension curve is at a peak), the tendon slack length (the length at which the tendon begins to assume load), and two global gain factors for flexion and extension. Physiologically based parameters are allowed to vary within one standard deviation of the literature-reported values (23); other parameters are bounded by stability criteria (2).

Experimental procedures. EMG were collected from three subjects using surface electrodes for the tibialis anterior, medial gastrocnemius, and lateral gastrocnemius, and fine wire intramuscular electrodes were used for the soleus. All subjects gave written informed consent and the protocol was approved by our human subjects review board. A telemetered system (Noraxon 9000) was used to transfer 

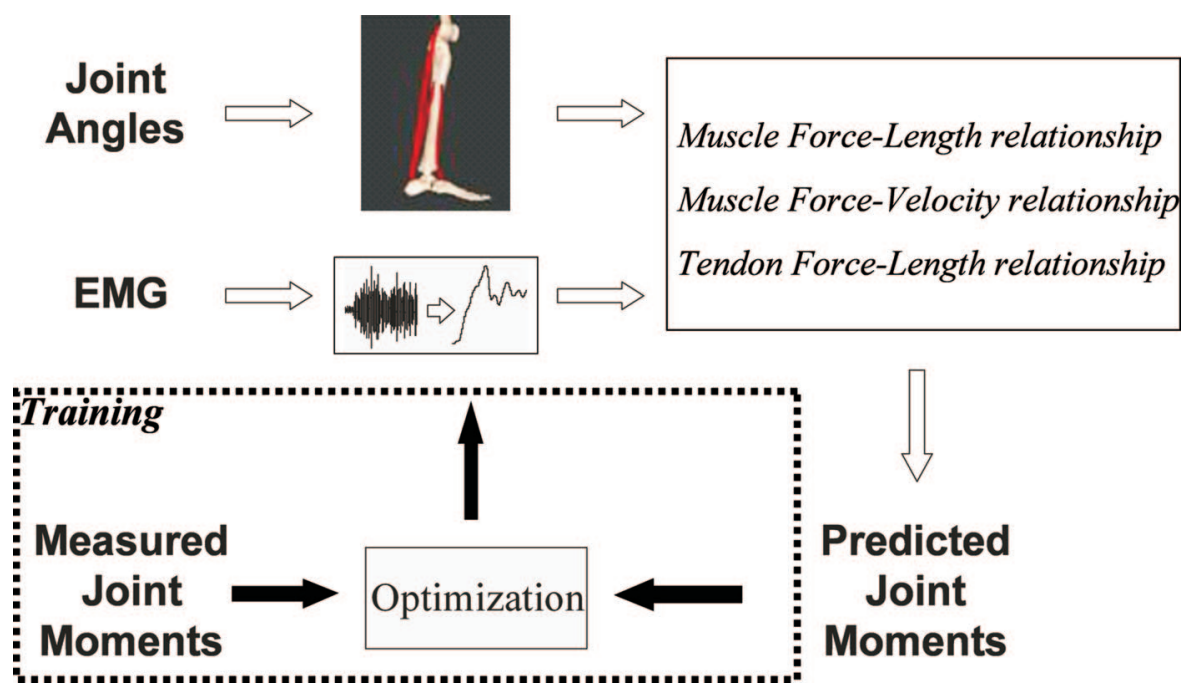

FIGURE 2-Simplified flowchart of the modeling procedure. The EMG is processed to obtain muscle activation, whereas the joint angle data are used to obtain musculoskeletal lengths, velocities, and moment arms. Together these are put into a Hill-type model to estimate musculotendon forces, and these, in turn, are multiplied by their moment arms and summed to obtain the joint moment. In the training phase (dotted box), the estimated moment is compared with the measured moment. Parameters in the model are adjusted to minimize the difference between measured and predicted joint moments. One the optimal parameters are found and the model is tuned for the subject, the training part of the model is removed; it can be used to predict joint moments for novel tasks.

the EMG signals to a custom-made amplifier with band pass filtering of $30-500 \mathrm{~Hz}$. The signals were then digitally sampled at $1 \mathrm{kHz}$.

EMG were collected during two types of trials: those performed in a dynamometer, and those during gait. Dynamometer trials included isokinetic concentric trials at 60, 120, and $150^{\circ} \cdot \mathrm{s}^{-1}\left(150^{\circ} \cdot \mathrm{s}^{-1}\right.$ is the maximum value for ankle angular velocity in the stance phase). The range of motion in the isokinetic trials was $5^{\circ}$ dorsiflexion to $20^{\circ}$ plantar flexion, which is the average range of motion of the ankle joint during gait. Preliminary data were collected during maximum voluntary contractions for normalization purposes.

To collect data during gait trials, we used a Qualisys motion capture system, using six Pro-reflex cameras, and an AMTI force plate. Ground reaction forces and spatial positions of the foot, shank, and thigh were calculated using a force plate and the motion analysis system. The ankle moment was calculated using inverse dynamics. Subjects were instructed to simply walk at a self-selected pace.

\section{RESULTS}

In this study, EMG were collected from four muscles and the resulting joint moments were estimated. The parameters for the model were then adjusted to minimize the difference between the estimated moments (from forward dynamics) and the measured moments (from inverse dynamics) (Fig. $3)$. The root mean squared error was $1.4 \mathrm{~N} \cdot \mathrm{m}\left(\mathrm{R}^{2}=0.997\right)$.

The model was also used to predict the moments during a novel task. Without changing the parameters of the model, the EMG and joint angles from a novel gait trial were input to the model, and the corresponding moment was predicted and compared with the measured moment determined using inverse dynamics (Fig. 4). The root mean squared error was $7.1 \mathrm{~N} \cdot \mathrm{m}\left(\mathrm{R}^{2}=0.94\right)$. The corresponding muscular forces are an intermediate step in the model (Fig. 5).

\section{DISCUSSION}

We have presented a model to estimate joint moments from EMG signals using a hybrid forward-inverse dynamic approach. This approach uses a Hill-type model that accounts for force-length and force-velocity relationships. The model results are verified by comparing the predicted joint moments with the measured moments.

These models have tremendous importance in estimating muscle forces during various tasks-something that is difficult to achieve with other modeling approaches. For ex-

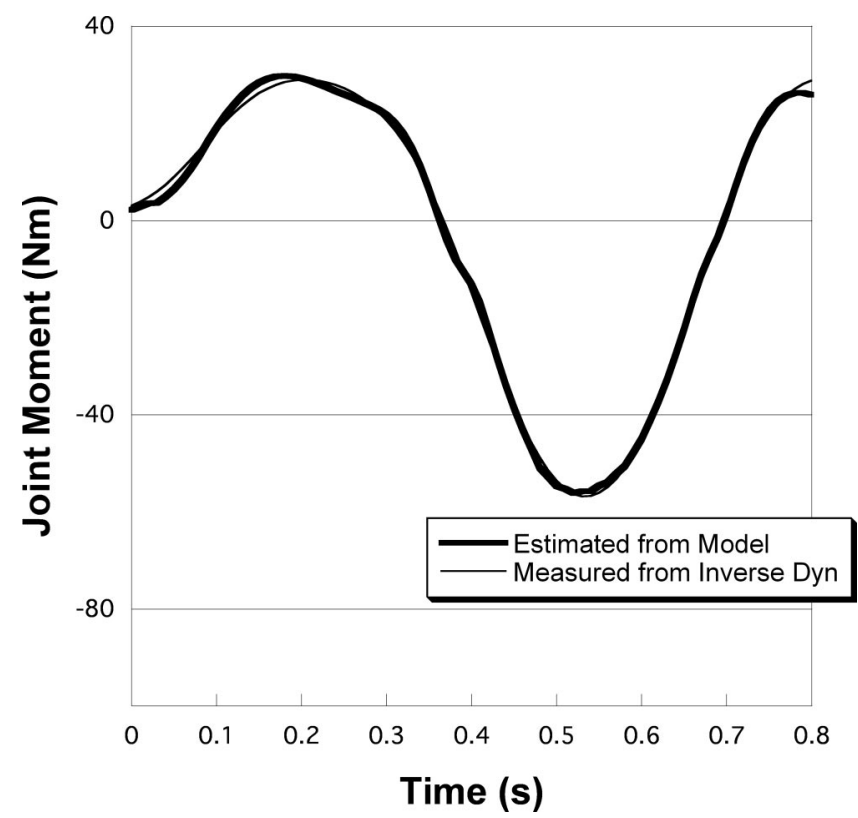

FIGURE 3-The model's ability to estimate ankle joint moments immediately after training on a concentric trial on a Biodex dynamometer. The model parameters were adjusted so that the error between the estimated and measured moments was minimized. Note that although the match is good, the number of parameters adjusted to achieve this fit is relatively low. 


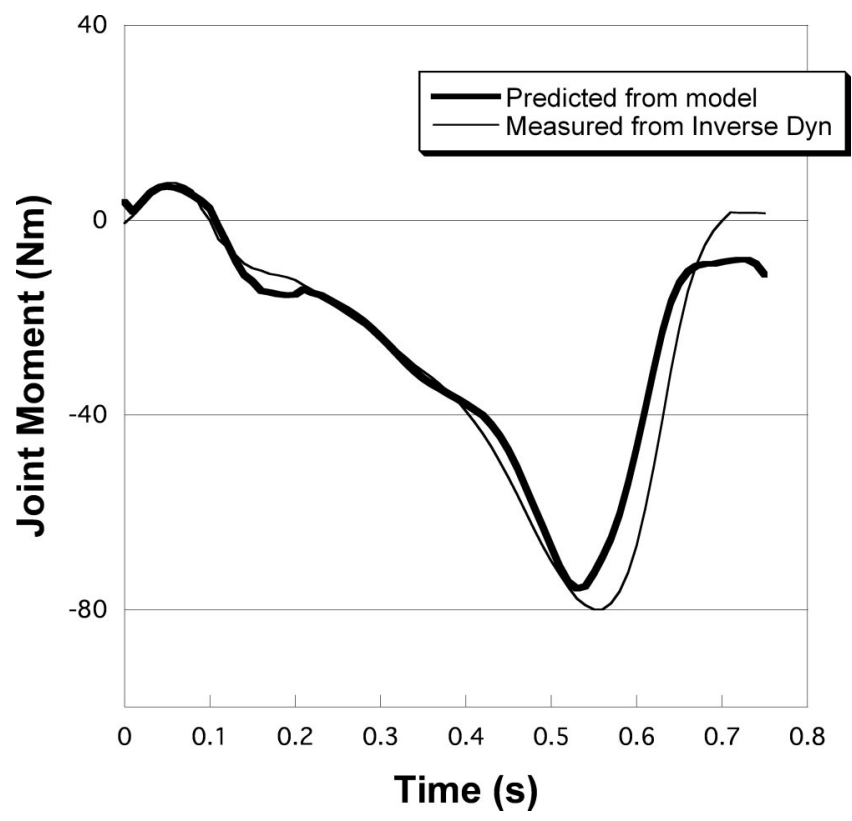

FIGURE 4-The model's ability to predict ankle joint moments during gait. Positive joint moments correspond to dorsiflexion. In this case, the parameters of the model were adjusted using data from a different trial, and then the model was used predict the moments in this gait trial. This demonstrates the model's ability to predict moments from a novel task using only EMG and kinematic information (i.e., without parameter readjustment).

ample, optimization-based models may be able to predict forces, but they do not readily account for differences in an individual subject's neuromuscular control system, which may be impaired.

The accuracy of this approach is greatly influenced by the accuracy and completeness of the anatomical data, which

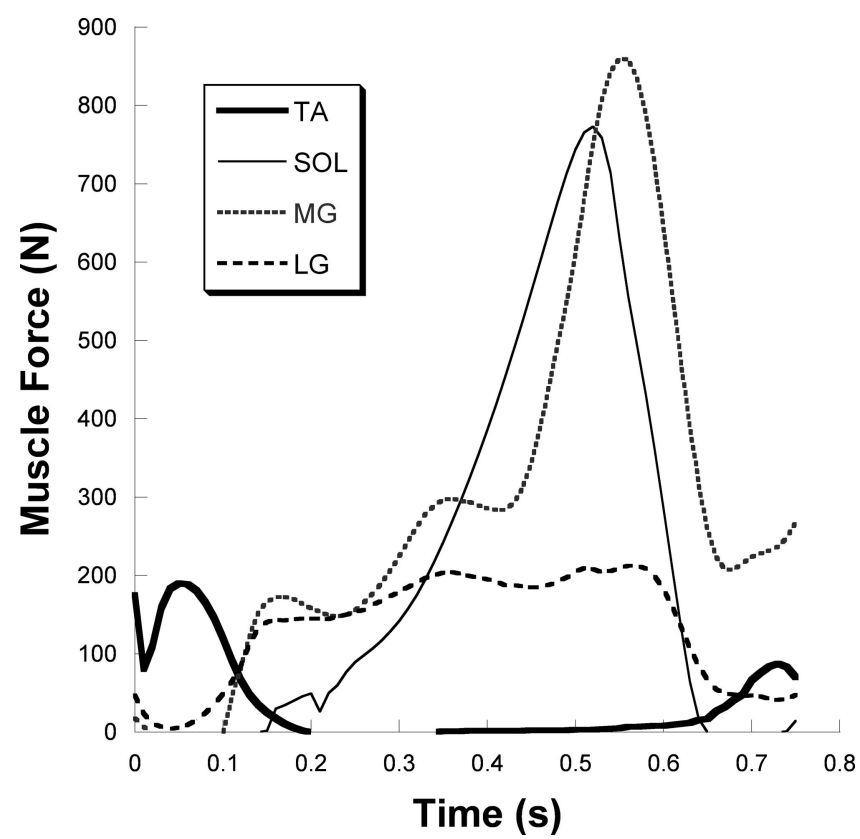

FIGURE 5-The force contribution of each muscle to the total joint moment. The muscle forces are multiplied by their respective moment arms and summed to yield the predicted moment in Figure 4. The muscles shown are the tibialis anterior (TA), medial gastrocnemius (MG), lateral gastrocnemius (LG), and soleus (SOL). must include a full model of the musculoskeletal geometry. In this example, a simple model of the ankle was used with only four muscles and a single degree of freedom. More elaborate models have been developed at other joints. For example, Lloyd and Besier (11) used 13 muscles in their model of the knee, and Manal et al. (16) used seven muscles in their model of the elbow. The error in the predictions of joint moment will be related to the completeness of the model, and this model of the ankle yielded errors of around $7 \mathrm{~N} \cdot \mathrm{m}$ in predicting joint moments during gait, which had peak ankle moments of around $80 \mathrm{~N} \cdot \mathrm{m}$. By comparison, the more detailed model of Lloyd and Besier (11) demonstrated errors of around $12 \mathrm{~N} \cdot \mathrm{m}$ during more demanding running and cutting tasks that had peak knee moments of around $200 \mathrm{~N} \cdot \mathrm{m}$.

The more parameters that are allowed to vary, the better the fit will be between the estimated joint moment and the measured joint moment. However, that does not mean that it is best to vary as many parameters as possible (9). Models that have many parameters generally have little predictive ability. Zheng et al. (25) created a model that estimates muscle forces from EMG, and it yields predicted joint moments that are very close to those determined using inverse dynamics. But their model requires that the parameters are determined or reevaluated at each instant in time. That is, the model is accurately adjusted to fit the data at each time step. But with many time steps, and perhaps hundreds or thousands of parameters to adjust during the course of a single movement, it is not unlikely that accurate predictions could be made. The problem with this approach is that the model is "overfit." This means that the model cannot be used in any predictive way. If parameters must be recalculated or adjusted for each trial, the model cannot be used to predict novel data. Although this may not have been a problem for Zheng et al.'s application, it means that such models will have limited predictive power, especially when used to predict a variety of different tasks such as walking, jogging, and jumping.

A predictive model is one that can be calibrated with some data and appropriate parameters adjusted within reasonable amounts. Those parameters that correspond to physically established measurements should not be allowed to be adjusted beyond physiological norms. Then, once the parameters are adjusted, the model can be used with novel data without further adjustment of the parameters. In this way, the robustness of the model's ability to predict the correct answers can be ascertained.

The advantage of the hybrid approach presented here is that the joint moments from the inverse solutions can be used to cross validate the forward modeling solutions, of course within the error associated with inverse dynamic methods. The calibrated forward model produced good predictions of the inverse solution of the ankle flexion-extension moments. Similar results have been reported for studies of the knee (11) and elbow (16) using this approach. In the Lloyd and Besier study (11), it was found that by keeping muscle-tendon parameters constant and only permitting the EMG-to-activation parameters to be adjusted, the model 
was able to predict trials $2 \mathrm{wk}$ apart with no loss in predictive ability. Once the model was calibrated and shown to be able to predict joint moments very well, they had confidence in the estimates of muscle forces and joint moments. They were then able to use the calibrated model to estimate loads experienced by the ligaments in the knee during the tasks performed by the subjects.

This modeling method is currently being used to determine ligament and joint compressive forces in walking, running, and cutting maneuvers. (Joint contact forces

\section{REFERENCES}

1. Anderson, F. C., and M. G. Pandy. Dynamic optimizationof human walking. J. Biomech. Eng. 123:381-390, 2001.

2. Buchanan, T. S., D. G. Lloyd, K. Manal, and T. F. Besier. Neuromusculoskeletal modeling: estimation of muscle forces and joint moments and movements from measurements of neural command. J. Appl. Biomech. 20:367-395, 2004.

3. Buchanan, T. S., M. J. Moniz, J. P. Dewald, and W. Zev Rymer. Estimation of muscle forces about the wrist joint during isometric tasks using an EMG coefficient method. J. Biomech. 26:547-560, 1993.

4. Corcos, D. M., G. L. Gottlieb, M. L. Latash, G. L. Almedia, and G. C. Agarwal. Electromechanical delay: An experimental artifact. J. Electromy. Kines. 2:59-68, 1992.

5. Delp, S. L., and J. P. LoAn. A graphics-based software system to develop and analyze models of musculoskeletal structures. Comput. Biol. Med. 25:21-34, 1995.

6. Delp, S. L., J. P. Loan, M. G. Hoy, F. E. Zajac, E. L. Topp, and J. M. Rosen. An interactive graphics-based model of the lower extremity to study orthopaedic surgical procedures. IEEE Trans. Biomed. Eng. 37:757-767, 1990.

7. Guimaraes, A. C., W. Herzog, T. L. Allinger, and Y. T. Zhang. The EMG-force relationship of the cat soleus muscle and its association with contractile conditions during locomotion. J. Exp. Biol. 198(Pt 4):975-987, 1995.

8. Heckathorne, and CW, D. S. Childress, Relationships of the surface electromyogram to the force, length, velocity, and contraction rate of the cineplastic human biceps. Am. J. Phys. Med. 60:1-19, 1981.

9. Heine, R., K. Manal, and T. S. Buchanan. Using Hill-type muscle models and EMG data in a forward dynamic analysis of joint moment: evaluation of critical parameters. J. Mech. Med. Biol. 3:169-186, 2003.

10. Hill, A. V. The heat of shortening and the dynamic constants of muscle. Proc. Royal Soc. London B. 126:136-195, 1938.

11. Lloyd, D. G., and T. F. Besier. An EMG-driven musculoskeletal model to estimate muscle forces and knee joint moments in vivo. J. Biomech. 36:765-776, 2003.

12. Lloyd, D. G., and T. S. Buchanan. A model of load sharing between muscles and soft tissues at the human knee during static tasks. J. Biomech. Eng. 118:367-376, 1996.

13. Lloyd, D. G., and T. S. Buchanan. Strategies of muscular support of varus and valgus isometric loads at the human knee. J. Biomech. 34:1257-1267, 2001. are different than the ground reaction forces determined from inverse dynamics.) This holds great promise as a method for studying in vivo tissue forces during sports and exercise.

This work is supported, in part, by NIH grants R01-AR46386, R01-HD38582, and P20-RR16458, as well as from the Australian NHMRC-991134 and NHMRC-254565, West Australian MHRIF, and AFL Research and Development Board. We thank Shay Cohen for his technical assistance with the data acquisition and ankle model development.

14. Manal, K., and T. S. Buchanan. A one-parameter neural activation to muscle activation model: estimating isometric joint moments from electromyograms. J. Biomech. 36:1197-1202, 2003.

15. Manal, K., and T. S. Buchanan. Subject specific estimates of tendon slack length: a numerical method. J. Appl. Biomech. 20: 195-203, 2004.

16. Manal, K., R. V. Gonzalez, D. G. Lloyd, and T. S. Buchanan. A real-time EMG-driven virtual arm. Comput. Biol. Med. 32:25-36, 2002.

17. McGill, S. M., and R. W. Norman. Partitioning of the L4-L5 dynamic moment into disc, ligamentous, and muscular components during lifting. Spine 11:666-678, 1986.

18. Olney, S. J., and D. A. Winter. Predictions of knee and ankle moments of force in walking from EMG and kinematic data. $J$. Biomech. 18:9-20, 1985.

19. Pandy, M. G., and F. E. ZaJac. Optimal muscular coordination strategies for jumping. J. Biomech. 24:1-10, 1991.

20. ScotT, S. H., and D. A. Winter. A comparison of three muscle pennation assumptions and their effect on isometric and isotonic force. J. Biomech. 24:163-167, 1991.

21. Thelen, D. G., A. B. Schultz, S. D. Fassois, and J. A. AshtonMilLER. Identification of dynamic myoelectric signal-to-force models during isometric lumbar muscle contractions. J. Biomech. 27:907-919, 1994.

22. Woods, J. J., and B. Bigland-Ritchie. Linear and non-linear surface EMG/force relationships in human muscles. An anatomical/functional argument for the existence of both. Am. J. Phys. Med. 62:287-299, 1983.

23. Yamaguchi, G. T., A. G. U. Sawa, D. W. Moran, M. J. Fessler, and J. M. WinTERS. A survey of human musculotendon actuator parameters. In: Multiple Muscle Systems: Biomechanics and Movement Organization, J. M. Winters and S. L. Woo (Eds.). New York: Springer-Verlag, 1990, pp. 717-773.

24. ZAJAC, F. E. Muscle and tendon: properties, models, scaling, and application to biomechanics and motor control. Crit. Rev. Biomed. Eng. 17:359-411, 1989

25. Zheng, N., G. S. Fleisig, R. F. Escamilla, and S. W. Barrentine. An analytical model of the knee for estimation of internal forces during exercise. J. Biomech. 31:963-967, 1998.

26. Zuniga, E. N., and E. G. Simons. Nonlinear relationship between averaged electromyogram potential and muscle tension in normal subjects. Arch. Phys. Med. Rehabil. 50:613-620, 1969. 